\title{
Access to substance abuse treatment ser- vices for black South Africans: findings from audits of specialist treatment facilities in Cape Town and Gauteng
}

\author{
Bronwyn Myers ${ }^{1} \&$ Charles DH Parry ${ }^{1,2}$ \\ Alcohol and Drug Abuse Research Group, Medical Research Council Cape Town, South Africa \\ Department of Psychology, University of Stellenbosch, Stellenbosch, South Africa
}

\begin{abstract}
Background: The increased demand for substance abuse treatment has led to concern about the accessibility of existing services to black South Africans. To date, research has not examined the accessibility of services, even though access has been shown to impact on retention and treatment outcomes. Method: Cross-sectional audits of substance abuse treatment facilities were conducted in Cape Town (2002) and Gauteng (2003). Data on client characteristics, facility characteristics, and service delivery characteristics were collected using the Treatment Services Audit questionnaire. Aims: To describe the extent to which substance abuse treatment services are accessible to black clients and the extent to which facilities target barriers that restrict black clients from accessing substance abuse treatment. Results: At both sites, black clients are under-represented at treatment facilities. Private non-profit, outpatient facilities serve the highest proportion of black clients. Compared to private for-profit and state facilities, private non-profit facilities are the most likely to provide services that address the logistical, cultural and linguistic barriers that restrict black clients from accessing treatment. Outpatient facilities are more likely than inpatient facilities to address these barriers. Conclusions: Based on the above findings, a number of recommendations are made to improve the accessibility of treatment services for black clients, such as establishing state outpatient treatment facilities and addressing the indirect costs associated with treatment.
\end{abstract}

Keywords: Treatment services, Substance abuse treatment, Access

Received: $31 / 08 / 2004$

Accepted: $28 / 09 / 2004$

Although there has been a growing demand for effective, affordable, and accessible substance abuse treatment services ${ }^{1}$, several socio-political factors have hampered access to treatment in South Africa*. Not only has funding to state-subsidised treatment services generally been inadequate, but under the apartheid system of governance, the available treatment facilities were poorly distributed, with services being concentrated in urban areas that were historically reserved for whites ${ }^{\#}$.

\footnotetext{
* Access to treatment refers to the extent to which entry into and retention in the substance abuse treatment system is facilitated ${ }^{2}$

\# The terms "white, black, asian/indian, and coloured" refer to demographic markers and do not signify inherent characteristics. These markers were chosen for their historical significance. These markers are important as accurate user profiles assist in identifying vulnerable sections of the population and in planning effective intervention programmes.
}

\section{Correspondence:}

Ms Bronwyn Myers, Alcohol and Drug Abuse Unit, Medical Research Council, Cape Town, PO Box 19070, Tygerberg, 7505, South Africa email:bronwyn.myers@mrc.ac.za
Major disparities also existed between the racially defined population groups in terms of the allocation of resources to, and the quality of substance abuse treatment services. ${ }^{3}$ Substance abuse treatment services were therefore not readily available to all sectors of the population. ${ }^{4}$

Since the country's transition to democracy in 1994, the health and social service sector has worked hard to improve service delivery to historically underserved groups, however concerns remain about the accessibility of substance abuse treatment services for black South Africans. In particular, there are concerns about the limited number of affordable facilities, especially given that there are few dedicated state-funded substance abuse treatment facilities in the country and that the number of beds available in state hospitals for substance use disorders has been reduced. Although there are statesubsidised treatment facilities, over time funding to these facilities has decreased in real terms, limiting their treatment capacity and their capacity to expand services to historically underserved areas. These reductions in funding have been a partial attempt to increase the accessibility of treatment ser- 
vices to historically underserved groups through shifting from providing mostly tertiary level services to integrating services into existing primary health care networks. While some steps have been taken to introduce services into primary and secondary levels of care, implementation has been slow, with few substance-related services being offered at the primary level.

In addition, the increased demand for substance abuse treatment 1 and the limited availability of state services has given rise to a growing private, for-profit treatment sector. Whilst these facilities fill an important gap in the market, they have been criticised for serving mostly white communities; having limited skills for dealing with the socio-cultural and language context of historically disadvantaged communities; being located in urban areas and consequently being inaccessible to the majority of the population; and for being largely unaffordable to those without medical aid. ${ }^{5}$

Despite concern regarding the accessibility of substance abuse treatment, and the awareness that effective service planning requires quality information about local needs, existing services, and patterns of service utilisation; ${ }^{6}$ there has been little research focusing on the accessibility of substance abuse services to black South Africans. This study begins to redress this gap through describing (i) the extent to which substance abuse treatment services are accessible to black clients and (ii) the extent to which facilities target barriers that prevent black clients from accessing substance abuse treatment.

\section{Methods}

\section{Study Design}

Separate cross-sectional surveys of substance abuse treatment facilities were conducted in Cape Town (August to November 2002) and in Gauteng Province (August 2003 to January 2004).

\section{Sample}

The sample consisted of all facilities in Cape Town $(\mathrm{N}=25)$ and Gauteng $(\mathrm{N}=36)$ that satisfied the criteria used by this study for the definition of a "specialist substance abuse treatment facility". In Cape Town, the response rate was $88.0 \%(\mathrm{~N}$ $=22$ ). The corresponding response rate for Gauteng was $86.1 \%$ $(\mathrm{N}=31)$. For both audits, non-responding facilities tended to serve fewer clients, be privately owned, have fewer staff and resources, and be in rural areas.

\section{Questionnaire}

The Treatment Services Audit (TSA) Questionnaire was used in Cape Town, and a revised version of the TSA was used in Gauteng, to collect self-report information from key informants at specialist treatment facilities. The construction of the original TSA was based loosely on the Unified Facility Data Set Questionnaire ${ }^{7}$ that has been used to collect one-day census information on the population of substance abuse treatment facilities in the USA. The questions contained in the original version of the TSA were discussed in focus groups of substance abuse treatment experts to ensure applicability to the South African context. The original TSA was then piloted at two treatment facilities in Cape Town and necessary changes were made to problematic items. Both versions of the TSA include structured questions with forced choice responses, as well as open-ended questions, and take about 45 minutes to complete. The TSA collects information from sev- eral domains, including: treatment facility characteristics, service delivery characteristics, types of treatment services offered, services to improve access to and retention in treatment, characteristics of clients served, staffing characteristics, organisational environment, and monitoring and evaluation activities.

\section{Data collection procedures}

For both audits, treatment programme managers and/or facility directors from all facilities in the sampling frame were contacted telephonically, informed about the study, and asked to participate. Informants were assured that identifying information on treatment facilities would not be included in the final reporting of data. Data collection packets, including the TSA, a guideline for completion of the TSA, and a covering letter explaining the purpose of the audit were sent via mail, fax, or email to the identified informants at participating facilities. During the data collection phase, the principal investigator was available to answer facilities' questions about the audit. Four weeks after the initial mailing, reminder letters were sent to all facilities and reminder telephone calls were made. Approximately eight weeks after the initial mailing, further reminder telephone calls were made to non-responding facilities. These facilities were also sent a second mailing. About four weeks after the second mailing, nonrespondents received a third reminder telephone call. Those respondents that had not responded within four weeks after the third reminder call, were telephoned again and sent a third mailing.

\section{Data analysis}

Descriptive statistics were calculated for all treatment service-, client-, staff-, and access-related variables. Facilities were stratified by treatment intensity and ownership. For each of these strata, cross-tabulations were performed on all variables. Chi-square tests of association were performed to determine if there were significant differences between the types of facilities on these variables.

\section{Results}

Demographic profile of clients at specialist substance abuse treatment centres in Cape Town and Gauteng

Compared to the demographic profile of the general populations ${ }^{8}$ in Cape Town and Gauteng, black South Africans were underrepresented and white South Africans were overrepresented in each of the respective treatment populations (Figure 1).

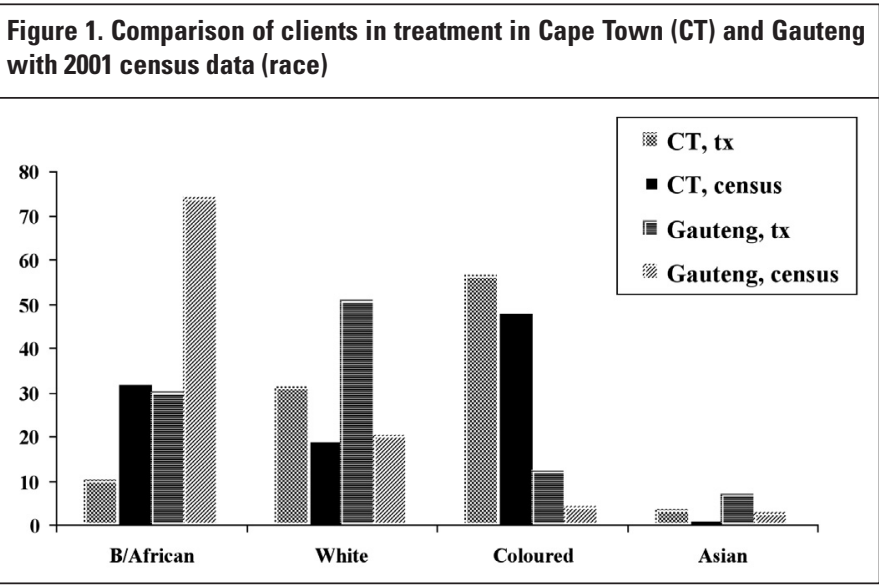


Table 1: Comparison of race profile of clients by facility ownership and treatment intensity in Cape Town (CT) and Gauteng (G).

\begin{tabular}{|l|c|c|c|c|c|c|c|c|}
\hline & \multicolumn{2}{|c|}{ Private for profit } & \multicolumn{2}{|c|}{ Private non-profit inpatient } & \multicolumn{2}{c|}{ Private non-profit outpatient } & \multicolumn{2}{c|}{ State inpatient } \\
\cline { 2 - 9 } & CT & $G$ & CT & $G$ & CT & $G$ & CT & G \\
\hline Mean \% of black & 3.1 & 11.4 & 2.2 & 19.6 & 24.5 & 51.1 & 4.8 & 30.5 \\
Mean \% of coloured & 28.1 & 3.6 & 51.7 & 8.9 & 65.3 & 23.2 & 79.6 & 8.5 \\
Mean \% of asian & 1.9 & 4.3 & 0.0 & 9.4 & 1.5 & 12.5 & 1.1 & 3.5 \\
Mean \% of white & 66.8 & 82.1 & 46.1 & 62.2 & 12.1 & 18.7 & 14.0 & 57.5 \\
\hline
\end{tabular}

For both Cape Town and Gauteng, private non-profit outpatient facilities were significantly more likely to serve a higher proportion of black clients than state inpatient, private non-profit inpatient, or private for-profit facilities (Chi-Square $=20.06, \mathrm{p}=$ 0.003, Chi-square $=17.04, p=0.048$, respectively). In contrast, private for-profit facilities in Cape Town and Gauteng were significantly more likely to serve a higher proportion of white clients than state inpatient, private non-profit inpatient, or private for-profit facilities (Chi-square $=19.37, \mathrm{p}=0.004$; Chi-square $=21.16, \mathrm{p}=0.012$, respectively) (Table 1 ).

\section{Proportion of facilities in Cape Town and Gauteng} that target barriers to treatment access and retention At both sites, less than half of the facilities offer outreach services in the township areas and less than a third conduct street outreach among under-served groups. While more than $80 \%$ of facilities in Cape Town and Gauteng report offering reduced fees to clients, significantly fewer provide free treatment slots for poorer clients $(Z=-2.02, p=0.004 ; Z=-3.36, p=0.001$, respectively). Similarly, less than a third of facilities provide transport services to clients. Less than a third of facilities in Cape Town and less than two-thirds of facilities in Gauteng employ Africanlanguage speaking therapists to help address cultural and linguistic barriers to treatment entry. While almost two-thirds of facilities report providing multilingual treatment services and almost all facilities report employing staff from diverse ethnic backgrounds, further questioning revealed that most facilities only offer programme services and translated instruments in English or Afrikaans (Figure 2).
Compared to inpatient facilities, a significantly higher proportion of outpatient facilities conduct outreach in the township areas (Chi-square $=4.18, \mathrm{p}=0.041$; Chi-square $=3.18, \mathrm{p}=0.049$ in Cape Town and Gauteng, respectively). A higher proportion of outpatient facilities offer reduced fees to clients than inpatient facilities in Cape Town (Chi-square $=3.79, \mathrm{p}=0.049$ ). Similarly, a higher proportion of outpatient than inpatient facilities provide programme services in a number of languages (Chi-square $=8.70, p=0.003$; Chi-square $=6.42, p=0.011$ ) and employ African-language speaking therapists (Chi-square $=6.14, \mathrm{p}=0.013$; Chi-square $=5.36, \mathrm{p}=$ 0.032) in Cape Town and Gauteng, respectively (Table 2).

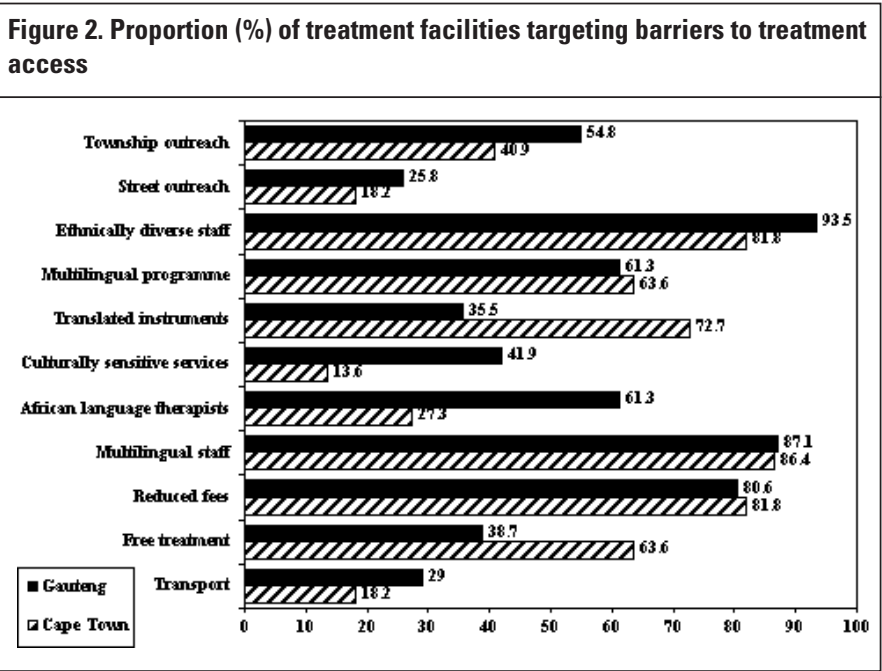

\begin{tabular}{|c|c|c|c|c|c|c|}
\hline & Site & Inpatient & Outpatient & Private for-profit & Private non-profit & State \\
\hline \multirow[t]{2}{*}{ Township outreach } & CT & 23.1 & 66.7 & 12.5 & 63.6 & 33.3 \\
\hline & Gauteng & 47.1 & 64.3 & 12.5 & 71.4 & 50.0 \\
\hline \multirow[t]{2}{*}{ Street outreach } & & 15.4 & 22.2 & 12.5 & 27.3 & 0.0 \\
\hline & Gauteng & 11.8 & 42.9 & 0.0 & 38.1 & 0.0 \\
\hline \multirow[t]{2}{*}{ African language- therapists } & & 7.7 & 55.6 & 12.5 & 45.5 & 0.0 \\
\hline & Gauteng & 57.1 & 64.7 & 25.0 & 71.4 & 100.0 \\
\hline \multirow[t]{2}{*}{ Multilingual staff } & & 76.9 & 100.0 & 75.0 & 100.0 & 66.7 \\
\hline & Gauteng & 85.7 & 88.2 & 100.0 & 50.0 & 100.0 \\
\hline \multirow[t]{2}{*}{ Reduced fees } & & 69.2 & 100.0 & 62.5 & 100.0 & 66.7 \\
\hline & Gauteng & 76.5 & 85.7 & 50.0 & 95.2 & 50.0 \\
\hline \multirow[t]{2}{*}{ Free treatment } & & 36.4 & 88.9 & 37.5 & 90.9 & 100.0 \\
\hline & Gauteng & 14.3 & 58.8 & 25.0 & 38.1 & 100.0 \\
\hline \multirow[t]{2}{*}{ Culture sensitive assessment and therapy } & & 0.0 & 33.3 & 0.0 & 100.0 & 0.0 \\
\hline & Gauteng & 41.2 & 42.9 & 25.0 & 66.7 & 50.0 \\
\hline \multirow[t]{2}{*}{ Translated materials } & & 53.8 & 100.0 & 25.0 & 100.0 & 100.0 \\
\hline & Gauteng & 29.4 & 42.9 & 12.5 & 47.6 & 0.0 \\
\hline \multirow[t]{2}{*}{ Multilingual programme } & CT & 38.5 & 100.0 & 25.0 & 90.9 & 66.7 \\
\hline & Gauteng & 58.8 & 64.3 & 12.5 & 76.2 & 100.0 \\
\hline
\end{tabular}


Compared to other types of facilities, a significantly lower proportion of private for-profit facilities conduct outreach in the township areas (Chi-square $=5.09, \mathrm{p}=0.038$; Chi-square $=8.14, \mathrm{p}=$ 0.017 in Cape Town and Gauteng, respectively). A higher proportion of private non-profit facilities offer reduced fees to clients than either state or private for-profit facilities (Chi-square $=4.91, \mathrm{p}=$ 0.046; Chi-square $=8.88, \mathrm{p}=0.012$ in Cape Town \& Gauteng, respectively). A lower proportion of private for-profit facilities offer free treatment to clients than other types of facilities (Chi-square $=$ 7.96, $\mathrm{p}=0.019$; Chi-square $=13.20, \mathrm{p}=0.001$ in Cape Town and Gauteng, respectively). Compared to private non-profit and state facilities, a lower proportion of private for-profit facilities in Cape town and Gauteng provide programme services in a number of languages $($ Chi-square $=8.71, \mathrm{p}=0.013$; Chi-square $=11.26, \mathrm{p}=$ 0.004 , respectively) (Table 2).

\section{Discussion}

Concerns about the availability, affordability, and accessibility of treatment services in South Africa, to a large extent, appear to be justified. Findings from this study show that the race profile of clients at specialist treatment facilities in Cape Town and Gauteng does not reflect the demographics of the general population. ${ }^{8}$ These findings are similar to those found by the South African Community Epidemiology Network on Alcohol and Drug Abuse (SACENDU) Network, which reported that black clients were significantly underrepresented in specialist substance abuse treatment centres between 1997 and 2001. ${ }^{1}$ Instead of reflecting lower levels of substance abuse, this pattern of service utilization probably reflects the limited extent to which treatment services are accessible to black South Africans, given that these facilities generally fail to address some of the common barriers that restrict black clients from accessing, engaging, and being retained in treatment. These barriers include: cultural and linguistic difficulties in participating in English (or Afrikaans) medium programmes, the stigma associated with seeking treatment for substance use disorders, and logistic factors such as the affordability of services and difficulties in paying for transport to facilities located in urban centres. ${ }^{1}$

More specifically, research has identified a lack of awareness of substance abuse treatment options and stigma associated with seeking treatment as barriers that restrict clients from seeking treatment. ${ }^{9}$ Although evidence suggests that outreach to improve knowledge around substance use disorders and awareness of treatment options significantly reduces stigma of treatment for under-served groups ${ }^{10}$, only a small proportion of facilities in Cape Town and Gauteng conduct activities that target these barriers among black South Africans.

Similarly, few treatment facilities perform activities that address the logistical barriers (such as transport and treatment costs) that prevent black clients from accessing and being retained in treatment. ${ }^{9,11}$ Facilities that do report addressing practical barriers to accessing care, are more likely to address financial concerns relating to the direct costs of treatment than other practical barriers, such as transport. For example, although most facilities offer reduced fees to clients, less than a third of facilities provide clients with transport services. Facilities should give serious consideration to ways in which the practical barriers and indirect costs associated with treatment, such as transport and distance required to travel to treatment, can be addressed for black South Africans, as addressing these barriers has been shown to significantly improve treatment retention. ${ }^{9,11}$

In addition, few facilities provide services that target the cul- tural and linguistic barriers that prevent black clients from accessing and being retained in substance abuse treatment. Although a high proportion of facilities report employing multilingual staff and staff from ethnically diverse backgrounds, further questioning revealed that for the most part, staff are fluent in English and Afrikaans only, and are white, coloured or asian/indian. This is confirmed by the finding that less than two-thirds of facilities employ African language speaking therapists. Similarly, less than two-thirds of facilities offer multilingual programmes. Of concern is the finding that less than half of the facilities use culturally sensitive and appropriate assessment and therapeutic approaches. These factors may not only inhibit black clients from seeking treatment, but may also impact on the extent to which black clients engage in treatment, with black clients being more likely to seek treatment at facilities which actively address the logistical and cultural/linguistic barriers they experience.

Organisational factors such as ownership and intensity of care are also associated with whether substance abuse treatment facilities in Cape Town and Gauteng target barriers to treatment for black clients, with outpatient facilities being more likely than inpatient facilities to target awareness-related, logistical, and cultural/linguistic barriers to entry and retention. Outreach activities (aimed at improving awareness of treatment options among black clients) are more likely to be conducted by outpatient than inpatient facilities, with a larger proportion of outpatient facilities conducting street outreach among vulnerable groups and outreach in townships than inpatient facilities. Outpatient facilities are also more likely to address logistical barriers to treatment than inpatient facilities, with a greater proportion of these facilities providing reduced fees and free treatment than inpatient facilities. Similarly, at both sites, outpatient facilities are more likely to address the cultural and linguistic barriers to treatment entry and retention for black clients, with a higher proportion of these facilities employing multilingual staff from diverse ethnic backgrounds, employing African-languagespeaking therapists, and offering a multilingual treatment programme than inpatient facilities. It is not surprising that outpatient facilities are more likely to address the cultural and linguistic barriers to treatment than inpatient facilities, as services at these facilities tend to be more affordable and geographically accessible than those provided by inpatient facilities. There may also be more pressure on outpatient facilities to provide culturally and linguistic appropriate services, given that they serve a greater proportion of black clients compared to inpatient facilities. However, it is also possible, that outpatient facilities serve significantly higher proportions of black clients because they actively target the barriers that restrict access to treatment for black South Africans.

Ownership status could also account for the difference between the proportion of black clients served at in-and outpatient treatment facilities. In South Africa, most outpatient treatment facilities have a private non-profit ownership status. In addition, ownership status is linked to whether treatment facilities in Cape Town and Gauteng target barriers to entry and retention, with private forprofit facilities being less likely to target awareness-related barriers to access and retention than private non-profit or state facilities. For example, at both sites, significantly fewer private for-profit facilities provide outreach services in the township areas than other types of facilities. Similarly, private for-profit facilities are less likely to address the logistical barriers to treatment entry and retention than other types of facilities, with private for-profit services being less likely to offer reduced fees and free treatment to clients than either private non-profit or state facilities. Private for-profit facili- 
ties are also less likely to address the cultural and linguistic barriers to treatment entry and retention for black clients, with a significantly lower proportion of these facilities employing multilingual staff from diverse ethnic backgrounds, employing African-language-speaking therapists, and offering a multilingual treatment programme than private non-profit or state facilities.

A possible explanation for these findings lies in the organisational goals of for-profit and non-profit facilities. Private for-profit facilities are likely to place more emphasis on the goal of profitmaximisation than other types of facilities. This goal could lead to organisations limiting their involvement in nonessential activities in order to reduce overhead costs. ${ }^{12}$ In addition, as private for-profit facilities are more likely to depend on private funding (such as health insurance or client self-pay fees) and less likely to receive state subsidies for treatment than private non-profit facilities, it may not be within the interests of these organisations to target clients that cannot afford the costs of treatment. As socio-economic status and race are still closely associated in South Africa, it is not surprising that private for-profit facilities are more likely to serve white than black clients. Furthermore, with a treatment population dominated by white and coloured clients, the lack of activities targeting treatment barriers for black clients should not just be interpreted as a deficit in services- especially given that there may not yet be a strong demand for culturally and linguistically-sensitive programmes at private for-profit facilities.

Based on this study's findings, several recommendations can be made regarding how the accessibility of substance abuse treatment services can be improved. As private, non-profit treatment facilities are the largest providers of treatment services to black clients, funding to these facilities should be increased. The establishment of state-funded outpatient facilities may be a means of providing cost-effective treatment services that are accessible to all sectors of the population. Awareness should also be raised among treatment providers about the importance of addressing barriers to treatment for black clients and the need for training in suitable methods of targeting these barriers. Logistical barriers to treatment should be addressed through increasing the number of beds available for impoverished clients and also through addressing the indirect costs associated with treatment. In addition, cultural and linguistic barriers could be addressed through offering multilingual treatment services and through employing African language speaking therapists. As facilities often struggle to find black/African substance abuse practitioners, a long term solution would be to encourage African language-speaking students of the health and other allied professions to enter the substance abuse field. Finally, interventions aimed at improving the cultural sensitivity of treatment programmes should be designed, implemented, and evaluated.

This study does, however, have a couple of methodological limitations. Given that this study has a cross-sectional design it cannot reveal whether patterns of service utilization among Black clients are shifting. Secondly although the response rate among treatment facilities was high, the sample size is still small and therefore the power to conduct statistical analyses is low. Finally, data derived from treatment settings in Cape Town and Gauteng are not representative of treatment settings in the rest of the country. These limitations suggest that we need to improve research efforts to describe the barriers to treatment entry for black South Africans by conducting focus group interviews of black clients receiving substance abuse treatment services and non-treated substance abusers. Furthermore, research needs to establish the extent to which black South Africans require accessible substance abuse treatment ser- vices, either through community-based needs assessments or through national household surveys. Finally, a regular, national audit of existing treatment centres in South Africa should be conducted, in order to monitor changes in the extent to which treatment facilities are accessible to black clients and the extent to which they attempt to address the barriers that restrict black clients from accessing treatment.

Despite these limitations, this study provides good evidence that access to treatment for black South Africans remains restricted by cultural/linguistic, logistic and awareness-related barriers. Substance abuse treatment facilities characterised by an outpatient intensity of care and private non-profit ownership status are not only more likely to address these barriers to treatment entry and retention, but are more likely to provide treatment services to black South Africans. The potential harm that untreated substance abuse holds for the individual and broader society, together with evidence of a causal relationship between treatment engagement, retention and client outcomes ${ }^{13}$ makes a strong case for the need for treatment service providers, researchers and policy-makers to address inequities in treatment service delivery and the barriers that restrict access to treatment for underserved groups.

\section{References}

1. Myers B, Parry CDH, Pluddemann A. Indicators of substance abuse treatment demand in Cape Town. Findings from the SACENDU project: 1998-2002. Curationis 2004;5: 27-31.

2. McCaughrin WC, Howard DL. Variation in access to outpatient substance abuse treatment: organisational factors and conceptual issues. J Subst Abuse Treat 1996; 8: 403-415.

3. Parry CDH, Bennetts A. Alcohol Policy and Public health in South Africa. Cape Town: Oxford University Press, 1998.

4. Parry CDH. Alcohol, drug abuse, and public health. In: Foster D, Freeman M, Pillay $Y$, eds, Mental health policy issues for South Africa. Cape Town: Medical Association of South Africa, 1997: 290-315.

5. Edelstein I, Weber V, Pillay Y. The role of the private sector. In: Foster $D$, Freeman M, Pillay $Y$, eds, Mental health policy issues for South Africa. Cape Town: Medical Association of South Africa, 1997: 132142.

6. Grant B, Petrie M. Alcohol and other drug treatment services. Development of a national minimum data set. Canberra: Australian Institute of Health and Welfare, 2001.

7. Carise D, McLellan AT, Gifford LS. Developing a national addiction treatment information system. Pennsylvania: Treatment Research Institute, 2002.

8. Statistics South Africa. The people of South Africa: population census, 2001. Census in Brief. Pretoria: Statistics South Africa, 2003.

9. Beardsley K, Wish ED, Fitzelle DB, O'Grady K, Arria AM. Distance travelled to outpatient drug treatment and client retention. J Subst Abuse Treat 2003; 25: 279-285.

10. Marsh JC, D'Aunno TA, Smith BD. Increasing access and providing social services to improve drug abuse treatment for women with children. Addiction 2000; 95 (8): 1237-1247.

11. Friedmann PD, Lemon SC, Stein MD. Transportation and retention in outpatient drug abuse treatment programmes. J Subst Abuse Treat 2001; $21:$ 97-103.

12. Friedmann PD, Alexander JA, D'Aunno TA. Organisational correlates of access to primary care and mental health services in drug abuse treatment units. J Subst Abuse Treat 1999; 16: 71-80.

13. Joe GW, Broome KM, Rowan-Szal GA, Dwayne Simpson D. Measuring patient attributes and engagement in treatment. J Subst Abuse Treat 2002; 22: 183-196. 